Service social

\title{
Les concepts de réseau et de crise comme fondements théoriques à l'intervention auprès de la personne âgée souffrant de maladie terminale
}

\section{Normand Gauthier}

Volume 34, numéro 1, 1985

Personnes âgées, milieux de vie et pratiques sociales

URI : https://id.erudit.org/iderudit/706255ar

DOI : https://doi.org/10.7202/706255ar

Aller au sommaire du numéro

Éditeur(s)

École de service social de l'Université Laval

ISSN

1708-1734 (numérique)

Découvrir la revue

Citer cet article

Gauthier, N. (1985). Les concepts de réseau et de crise comme fondements théoriques à l'intervention auprès de la personne âgée souffrant de maladie terminale. Service social, 34(1), 130-147. https://doi.org/10.7202/706255ar d'utilisation que vous pouvez consulter en ligne. 


\section{COMMENTAIRES \\ ET DOCUMENTS}

GAUTHIER, Normand, chargé de cours à l'École de service social de l'Université Laval.

\section{Les concepts de réseau et de crise comme fondements théoriques à l'intervention auprès de la personne âgée souffrant de maladie terminale}

\section{Normand Gauthier}

Même si la mort nous concerne tous, nous ne sommes pas tous familiers avec ce phénomène inévitable, surtout qu'elle ne se présente pas toujours de la même manière. Certaines personnes sont moins privilégiées que d'autres : par exemple, les cancéreux sont de plus en plus préoccupés par les conséquences physiques et psychosociales de leur maladie, préoccupation bien souvent doublée de certaines autres conditions contraignantes reliées à la problématique des personnes âgées. Car nous pouvons le dire : beaucoup de personnes relativement âgées sont atteintes d'une maladie terminale. Bien souvent, celle-ci se présente au moment ou à la veille de la mise à la retraite, étape représentant déjà un changement choc dans la vie d'une personne.

Ces malades ont, depuis plusieurs années, été confiés davantage au milieu hospitalier, les familles s'étant départies de leurs responsabilités et ayant mis de côté leurs aptitudes et leurs capacités à prendre en charge, elles-mêmes, le mourant. Tous ces éléments réunis ont donc contribué à amplifier cette situation de crise auprès du mourant et de ces familles (Saillant, 1982).

La famille est pourtant reconnue comme essentielle et irremplaçable auprès du mourant. C'est quand même elle qui, malgré un 
état de bouleversement, doit réagir le plus adéquatement possible à la suite d'un diagnostic, souvent inattendu, d'un cancer chez un proche. Considérant ses limites et ses forces, elle demeure alors potentiellement ouverte à de l'aide extérieure. Comme réseau d'aide, la famille se trouve donc à la fois dans une situation d'aidant et d'aidée, cette dualité étant souvent occultée dans sa seule dimension "aidée».

C'est pourquoi il faut redonner à la famille sa place privilégiée et favoriser son implication effective dans le processus du mourir d'un de ses proches. Celle-ci, tout comme le malade, pourrait être active et adéquate dans cette démarche. C'est d'abord à travers sa propre implication que chacun des membres voudra trouver un sens à cette épreuve. Cette démarche contribuera à aider la cellule familiale à liquider psychologiquement la perte qu'elle va subir.

Dans la littérature, on a déjà abordé la maladie physique comme situation de crise (Parad et Caplan, 1965). Mais, dans ce sens, la maladie terminale présente des réalités encore plus spécifiques pour les praticiens.

Les écrits sur l'intervention de réseau ont aussi traité de la crise (Daher, 1980). Mais l'intervention de réseau prend un sens plus caractérisé lorsque la situation-problème vise une personne âgée aux prises avec la maladie terminale.

Mes observations m'ont permis de réaliser ce fait. Lors d'une situation de crise provoquée par un diagnostic de maladie terminale, un réseau d'aide se mobilise spontanément pour satisfaire les besoins du malade autant que ses propres besoins. Mais une question se pose à ce moment-ci aux professionnels de l'aide (travailleurs sociaux, psychothérapeutes, médecins, infirmiers, etc.): comment ce réseau naturel d'aide peut-il être assuré de son efficacité? Une première démarche viserait à :

"fournir le plus d'information possible au professionnel sur les multiples facettes de l'aide naturelle, non seulement pour remédier à sa méconnaissance, mais surtout parce que l'actualisation de son nouveau rôle exige de lui qu'il connaisse très bien le monde de l'aide naturelle.» (Guay, 1984 : 3.)

À mon point de vue, cette connaissance engendrerait une meilleure collaboration entre intervenants naturels et professionnels. Et cela assurerait également une plus grande pertinence et complémentarité dans leurs rapports puisque notre rôle professionnel devra se baser fondamentalement sur un partage de la responsabilité avec les aidants naturels si certaines tâches peuvent être assumées par ceux-ci.

Cette réflexion est issue de ma propre expérience d'intervention auprès d'une personne âgée cancéreuse de mon entourage. Mais, 
comme travailleur social, j'ai recherché les fondements théoriques sous-tendant mes actions. Celles-ci s'appliquent donc ici à une intervention auprès d'une personne qui, à la veille de la mise à la retraite, fut aux prises avec un diagnostic de récidive du cancer.

Il importe maintenant de vous présenter ces fondements théoriques et je tiendrai compte principalement des éléments situationnels qui viennent ancrer l'intervention.

Le diagnostic subit d'un cancer: le diagnostic issu à un moment clé de la vie de quelqu'un, en l'occurrence une personne âgée, provoque définitivement une crise. D'où la nécessité de référer à une approche en situation de crise.

La gravité de l'événement: au moment précis du diagnostic, la personne ne peut répondre seule adéquatement à sa situation. De plus, la famille est à la fois directement concernée et ébranlée par la situation causée par la maladie terminale. C'est pourquoi celle-là et les ressources du milieu peuvent évidemment être impliquées dans un processus d'intervention. D'où la possibilité de privilégier une approche d'intervention de réseau. En premier lieu, on peut rappeler le principe qu'il existe dans notre société une importance et un potentiel de réseaux d'aide non professionnels (Burke et Weir, 1975). De plus, pour éviter toute confusion, il faut se souvenir que la maladie terminale n'est pas synonyme de phase terminale, mais plutôt qu'elle fait suite au diagnostic de la maladie et de la phase curative (Saillant, 1982). Ainsi, le champ d'intervention privilégié dans cette étude se détermine par la période fixée entre le diagnostic de récidive du cancer et la phase curative de la maladie.

Pour bien saisir la portée des deux approches de crise et de réseau, il nous faut tenir compte d'une méthodologie d'intervention appropriée à la condition de vie des personnes âgées. ${ }^{1}$

Dans un modèle d'intervention en gérontologie, tout le cadre théorique pour élaborer un diagnostic et fixer des objectifs est basé sur le concept de "réussite du vieillissement". La situation de maladie terminale et de mise à la retraite anticipée et non prévisible représente donc une difficulté certaine dans cette démarche fondamentale. Cette réussite se définit par un état d'esprit comportant un aspect cognitif et un aspect affectif, i.e. qu'il faut travailler sur la façon de vivre les événements et non de les changer. C'est là toute la particularité d'intervenir à ce niveau lorsqu'une personne âgée apprend le diagnostic brutal d'une maladie terminale. Il faut bien le dire, il est difficile de donner un sens positif à des événements perçus comme négatifs par la personne concernée par la maladie. Dans les faits, les activités de ce modèle produiront une certaine efficacité par la relation d'aide. Mais 
intervenir à quel niveau ? II y a plusieurs séquences d'activités proposées qui ne se présentent pas toujours de manière aussi exhaustive et ordonnée dans la réalité.

Pour bien faire saisir la dynamique de l'intervention, j'avais l'intention d'établir un tableau synoptique des séquences d'activités du modèle d'intervention gérontologique (tableau 1). Mais j'ai surtout voulu y mettre en lumière l'entrée en scène des différents fondements théoriques, inhérents à l'approche en situation de crise et à l'approche réseau, à l'intérieur même de ces séquences d'activités. Il décrit mon intervention auprès d'une personne âgée aux prises avec la maladie terminale.

Le tableau 1 n'a pas pour objectif de démontrer les phases d'intervention d'un soi-disant nouveau modèle approprié à la maladie terminale chez une personne âgée; faisant suite à mon expérience personnelle, j'ai simplement voulu y articuler une pensée logique autour d'un cadre d'intervention.

Associons maintenant une méthodologie d'intervention auprès de personnes âgées avec deux événements significatifs : la maladie grave et la mise à la retraite brutale et illustrons le parallèle existant en définissant la crise comme une période de déséquilibre que les mécanismes homéostatiques ordinaires de l'individu sont impuissants à résoudre. (Parad et Caplan, 1965 : 56.)

Donc, une crise est la résultante de certaines caractéristiques des circonstances qui la précipitent. Cette période critique se décrira alors comme étant un état de crise où les événements qui l'enclenchent requièrent une solution qui est nouvelle en regard des expériences vitales antérieures de l'individu. La mise à la retraite au moment même du diagnostic du cancer et la maladie fatale elle-même sont là autant de faits qui amènent le bouleversement d'un état, généralement stable, de la personnalité.

L'ensemble de ces faits dans la vie d'un individu ébranle sans contredit la manière dont celui-ci satisfait ses besoins fondamentaux (physiques, psychologiques et sociaux). C'est pourquoi il y a perturbation des manouvres constantes d'adaptation et des activités de type solution de problèmes auxquelles l'individu se raccroche. C'est ce que l'on appelle les mécanismes homéostatiques. Ceux-ci se révèlent donc inadéquats et ne peuvent rétablir rapidement l'état antérieur d'équilibre.

Pour mieux saisir cette réalité, on peut se référer à l'Annexe 1 qui retrace une méthodologie d'intervention en situation de crise.

Reprenons chacune des séquences d'activités en intégrant les fondements théoriques liés à la crise et au réseau. 


\section{TABLEAU 1}

Tableau synoptique de l'intervention gérontologique

\begin{tabular}{|c|c|c|c|c|}
\hline \multicolumn{2}{|c|}{ Intervention de crise comme fondement théorique } & \multirow{2}{*}{$\begin{array}{l}\text { Séquences } \\
\text { d'activités }\end{array}$} & \multicolumn{2}{|c|}{ Intervention de réseau comme fondement théorique } \\
\hline $\begin{array}{l}\text { Objectifs à } \\
\text { poursuivre }\end{array}$ & Le malade & & $\begin{array}{l}\text { Objectifs à } \\
\text { poursuivre }\end{array}$ & L'entourage \\
\hline $\begin{array}{l}\text { Prendre un contact rapide } \\
\text { et adéquat avec le malade } \\
\text { et la situation } \\
\text { Diminuer le stress situa- } \\
\text { tionnel et mesurer le } \\
\text { degré de désespoir de } \\
\text { la famille }\end{array}$ & $\begin{array}{l}\text { Explorer la nature et } \\
\text { l'étendue de la crise chez } \\
\text { le malade } \\
\text { Voir à la satisfaction de } \\
\text { ses besoins fondamentaux }\end{array}$ & 1 & $\begin{array}{l}\text { Rendre le réseau visible et } \\
\text { favoriser les contacts entre } \\
\text { ses membres } \\
\text { Planifier les disponibilités } \\
\text { des membres selon leur } \\
\text { potentialité } \\
\text { Participation effective des } \\
\text { ressources }\end{array}$ & $\begin{array}{l}\text { Établir un contact et favo- } \\
\text { riser une collaboration } \\
\text { étroite avec les gens con- } \\
\text { cernés par la maladie ter- } \\
\text { minale (famille, amis, voi- } \\
\text { sinage, médecins, etc.) }\end{array}$ \\
\hline $\begin{array}{l}\text { Centrer l'attention sur le } \\
\text { malade et la situation à } \\
\text { modifier } \\
\text { Viser, chez le malade, une } \\
\text { connaissance cognitive de } \\
\text { la situation }\end{array}$ & $\begin{array}{l}\text { Identifier le degré d'auto- } \\
\text { nomie du malade } \\
\text { Aider celui-ci à briser la } \\
\text { spirale descendante de la } \\
\text { perte d'autonomie psy- } \\
\text { chologique }\end{array}$ & II & $\begin{array}{l}\text { Mobiliser les membres } \\
\text { Identifier, avec le réseau, } \\
\text { des éléments de solution }\end{array}$ & $\begin{array}{l}\text { Supporter, préserver ou } \\
\text { restaurer l'autonomie } \\
\text { psychologique } \\
\text { Investir la mise en com- } \\
\text { mun des énergies vers la } \\
\text { solution de problème }\end{array}$ \\
\hline
\end{tabular}




\begin{tabular}{|c|c|c|c|c|}
\hline $\begin{array}{l}\text { Attribuer le crédit du } \\
\text { changement au malade } \\
\text { lui-même } \\
\text { Stimuler les énergies du } \\
\text { système-client et de sa } \\
\text { famille }\end{array}$ & $\begin{array}{l}\text { Assurer ou maintenir une } \\
\text { fonction stabilisatrice de } \\
\text { l'identité personnelle }\end{array}$ & III & $\begin{array}{l}\text { Fournir du support indi- } \\
\text { viduel } \\
\text { Faciliter les communi- } \\
\text { cations } \\
\text { Repérer les activités du } \\
\text { réseau }\end{array}$ & $\begin{array}{l}\text { Aider l'entourage à } \\
\text { supporter le processus de } \\
\text { développement de la } \\
\text { personnalité du malade } \\
\text { Favoriser leur propre } \\
\text { développement }\end{array}$ \\
\hline $\begin{array}{l}\text { Reconnaître les difficultés } \\
\text { rencontrées } \\
\text { Consolider les acquis } \\
\text { Ramasser et relancer les } \\
\text { compétences nouvelles }\end{array}$ & $\begin{array}{l}\text { Conserver ou restaurer le } \\
\text { mouvement de la vie } \\
\text { Travailler avec l'état } \\
\text { d'esprit du malade âgé } \\
\text { devant les conséquences } \\
\text { de la maladie terminale } \\
\text { Adapter les objectifs à } \\
\text { long terme dans une } \\
\text { perspective de court } \\
\text { terme }\end{array}$ & IV & $\begin{array}{l}\text { Reconnaitre les difficultés } \\
\text { rencontrées et fournir } \\
\text { encouragement au réseau } \\
\text { Favoriser et maintenir une } \\
\text { solidarité entre les } \\
\text { membres } \\
\text { Constitution d'unités } \\
\text { de travail } \\
\text { Relancer l'espoir }\end{array}$ & $\begin{array}{l}\text { Anticiper les difficultés et } \\
\text { les relier aux ressources } \\
\text { d'aide du réseau naturel } \\
\text { ou formel } \\
\text { Encourager et supporter } \\
\text { individuellement les } \\
\text { membres et favoriser leur } \\
\text { regroupement }\end{array}$ \\
\hline
\end{tabular}




\section{Première séquence d’activités}

Dans un premier temps, le travailleur social voit à la satisfaction des besoins (physiques, psychologiques, sociaux). Il établira une collaboration étroite avec le malade, sa famille immédiate et les effectifs médicaux, ainsi qu'entre toutes ces personnes. Cette concertation aura pour but premier de diminuer le stress situationnel. En tant qu'intervenants sociaux, nous devrions donc, au départ, nous interroger sur deux aspects de la situation. Ainsi, s'il y a lieu d'intervenir, est-ce que le réseau naturel ${ }^{2}$ suffit pour aider, d'une part la personne cancéreuse et, d'autre part, pour s'aider lui-même? Et, se basant sur une méthodologie d'intervention auprès d'une personne âgée cancéreuse, est-il approprié d'intervenir dans cette situation selon une approche en situation de crise?

Si oui, la réponse spontanée du réseau naturel se centrera sur les besoins concrets d'existence du malade et de sa famille. Un support organisationnel et fonctionnel s'établira selon les besoins propres à la situation et selon les habiletés et disponibilités de ses membres. La qualité de ces interventions dépendra primordialement de deux facteurs: du degré de capacité des membres du réseau naturel à réagir adéquatement dans des situations de stress intense et de l'accessibilité d'un support professionnel pour ceux-ci.

Mais au-delà des degrés relatifs aux facteurs précités, il y a une certitude sur laquelle je veux insister. Le travailleur social comme aidant professionnel peut intervenir de manière pertinente dans cette situation de diagnostic subit de maladie terminale. $\grave{A}$ un premier niveau, il aura un rôle tantôt plus impliqué, tantôt plus effacé auprès du malade et de sa famille, qui dépendra de l'autonomie démontrée par le réseau naturel d'aide. Dans ce partage de rôle, il pourra donc s'investir comme coordonnateur ou même stimulateur des énergies mises en commun. Tous participent à la recherche de solution pour pallier aux besoins entraînés par cette situation, tant au niveau du réseau que du malade lui-même. réseau :

Les critères suivants peuvent établir la pertinence de l'approche de

- la nature et l'étendue de la crise,

- le degré de désespoir de la famille,

- les efforts antérieurs faits pour résoudre le problème,

- la disponibilité suffisante de ressources chez la famille et les amis,

- la disponibilité suffisante des membres à une participation effective des ressources. 
On observe souvent que peu de membres immédiats d'une famille sont disponibles. Si la nature de la crise justifie la mise en place d'un réseau, il faut alors mobiliser les amis et leurs propres amis comme nouvelle alternative.

Car il y a effectivement crise dans le cas d'une maladie terminale. Pour le déterminer, il suffit de se baser sur les trois caractéristiques suivantes :

1. Le problème apparaît d'une importance fondamentale à la personne car il est relié à ses besoins fondamentaux ;

2. il ne peut être résolu rapidement par la personne avec ses propres mécanismes normaux de solution de problème;

3. entraîné par le diagnostic de cancer, il inclut une brève et intense période de stress, ces deux critères devant être présents simultanément pour justifier la crise.

L'intensité se détermine habituellement selon deux sources soient : la nature de l'événement stressant : d'une part, la maladie terminale est expérimentée comme une attaque au niveau de la satisfaction des besoins fondamentaux et, d'autre part, elle requiert une solution qui est nouvelle en relation avec l'expérience de vie antérieure; et le caractère subit de cet événement: il est vécu comme une brisure, la mort étant, à terme, la seule éventualité.

Quant à la brièveté de la crise, celle-ci découle elle-même de cette caractéristique d'intensité, c'est-à-dire l'impossibilité de vivre dans un état de stress intense très longtemps au détriment de la structure d'équilibre de la personnalité, et où l'intensité fait elle-même pression pour une solution rapide sans en remettre au lendemain.

\section{Deuxième séquence d'activités}

Dans cette seconde séquence, il s'agit de supporter, préserver ou restaurer l'autonomie psychologique du malade et de sa famille immédiate.

Nous faisons référence au concept de "lieu de contrôle", i.e. tabler sur ce que la personne voit d'elle-même. L'intervenant s'interrogera à savoir : est-ce que l'individu âgé, malade, se place en contrôle de sa vie interne, et quelle influence cela peut-il avoir sur ses proches? En d'autres mots, comment la personne voit-elle son rôle, elle qui est aux prises avec ces phénomènes extérieurs engendrés par la maladie terminale ? Concrètement, il s'agira d'impliquer activement le malade âgé dans la solution de sa situation-problème. C'est donc "l'aider à 
développer sa maîtrise cognitive et à briser la spirale descendante de la perte d'autonomie psychologique». (Laforest, 1983.)

Une phase importante de travail centrera le travailleur social et le malade sur la recherche des champs de préoccupation auxquels celuici est confronté par sa condition. Il y a, par exemple, plusieurs plans : l'entretien de la maisonnée, le travail, la pré-retraite, le domaine financier, le plan juridique et la santé. Par la suite, c'est en établissant avec lui un ordre de priorités que des membres du réseau pourront être investis comme support.

En résumé, après avoir identifié l'événement déclenchant, soit le diagnostic de cancer et les facteurs qui favorisent l'état de déséquilibre de l'individu, l'intervenant doit déterminer le degré de perturbation causé par la crise dans la vie de l'individu âgé cancéreux. Il le vérifie par des questions sur ses capacités à travailler, à tenir la maison, à prendre soin de sa famille, à prendre des décisions rationnelles et appropriées dans sa situation de maladie terminale.

En somme, les premiers points à considérer sont: dans quelle mesure ses activités sont-elles affectées ? comment cet état de déséquilibre affecte-t-il les personnes de son entourage? celles-ci sont-elles troublées et que pensent-elles qu'il devrait faire?

\section{Troisième séquence d'activités}

II va sans dire que de tels événements pourront même compromettre l'identité personnelle du malade. II faudra alors assurer le processus de développement de sa personnalité. Pour ce faire, il s'agit de maintenir une fonction stabilisatrice de l'identité personnelle. Cela se traduit par des interventions rendant disponibles des expériences de vie valides et même valorisantes. Comme exemple à l'appui, il faut savoir travailler sur les mécanismes de défense propres à la personne âgée aux prises avec une maladie terminale. Ainsi, une forme de rationalisation empêchera le malade de s'investir à court terme au niveau de ses traitements sous prétexte (justifiable bien souvent) de remplir ses rôles d'âge adulte : l'éducation de ses jeunes enfants, son rôle de pourvoyeur ou de gestion administrative de la maisonnée, etc. Cette attitude serait, d'une part, en opposition directe avec le caractère urgent de la situation et, d'autre part, nuirait à l'harmonie entre les trois composantes de l'identité personnelle (le moi connu, le moi idéal, et l'estime de soi) qu'il faut favoriser.

La famille immédiate est la plus directement touchée par la situation de maladie terminale. Chacun de ses membres, devant 
l'éventualité de la mort d'un de ses proches, se voit confronté avec sa propre mort. Le travailleur social aura donc un rôle particulier à jouer auprès de chacun dans cette étape de prise de conscience individuelle, ou même de croissance personnelle ; il pourra être conseiller, médiateur, facilitateur ou personne-ressource auprès des individus membres de la famille immédiate ou de l'ensemble de la famille. Les interventions individuelles et de groupe représentent donc des avenues possibles dans cette relation d'aide. Chacun des membres du réseau naturel et la famille dans son ensemble ont alors besoin de s'aider et d'être aidés pour surmonter cette expérience stressante et particulière.

\section{Quatrième séquence d'activités}

Il s'agit de conserver ou de restaurer le mouvement de la vie. C'est à cette étape que l'intervention du travailleur social se veut la plus délicate car cette dernière séquence devrait correspondre à des objectifs à plus long terme. Ceux-ci, malheureusement, sont limités par la dimension inéluctable du temps inhérente au cancer. Je donnerai cet exemple : un individu âgé s'était donné comme but de se permettre d'acheter une première auto neuve dans sa vie au moment de sa mise à la retraite. Un diagnostic de maladie terminale survient peu avant la retraite et compromet la réalisation de ce but. Mon rôle d'intervenant sera d'aider la personne à cheminer dans son choix et même de l'accompagner dans sa démarche.

Le choc de ce diagnostic modifie ainsi le vécu des malades: diminution des conditions de vie (baisse de revenu, capacité moindre de tenue de maison, etc.); perte d'autonomie physique (incapacité partielle de parler ou de se déplacer) et psychologique (difficulté de réfléchir adéquatement à la situation); attaque de son identité personnelle (se voir impuissant devant la maladie ou dépendant des autres); marginalisation dans ses conditions de vie ou mise à l'écart par ses proches.

C'est une situation imprévue constituant un problème important pour la personne et son entourage. D'autant plus sérieux qu'ils ne peuvent l'éviter ou que le malade n'est pas en mesure de résoudre la situation avec ses ressources d'aide habituelles. Il y a alors une période relativement courte de déséquilibre psychologique.

Devant les conséquences de la maladie terminale, l'intervenant devra adapter les objectifs à long terme, reliés au concept du mouvement de la vie, dans une perspective à court terme. Il faut aider la personne à retarder le déclin, sans pour autant renier les incapacités 
éventuelles puisque le déclin peut être accéléré avec l'évolution de la maladie. Cette qualité de l'intervention devra s'établir avec l'état d'esprit de la personne en situation; il faudra donc travailler à rendre accessible une possibilité de croissance individuelle puisqu'elle est toujours capable de vivre des expériences nouvelles.

En conclusion, pour poursuivre selon une approche de réseau, il faut se rappeler que l'intervention est faite à partir de la demande de la personne en crise. Nous considérons sa situation actuelle, sa dynamique familiale et souvent para-familiale, le changement souhaité et le niveau jusqu'où la personne peut aller. Ces données objectives et subjectives sont explorées en accord avec les actions préalables et privilégiées dans une intervention de réseau.

L'évaluation diagnostique et le contrat entraînent nécessairement deux activités qui permettent de réaliser ces buts : I'utilisation systématique des connaissances de praticien pour recueillir de l'information au sujet du client, de sa situation, et de sa famille ; l'utilisation consciente de soi-même par l'intervenant dans la recherche des difficultés et de leurs causes.

Dans une situation de crise, le client arrive en état de vulnérabilité, incapable de répondre adéquatement, incapable d'utiliser les mécanismes appropriés. Voilà pourquoi le réseau peut représenter le moteur central dans l'intervention.

La citation qui suit vient très bien résumer le sens des interventions, et elle rejoint celle d'autres auteurs tels que Speck et Attneave (1973).

"L'intervention de réseau de famille est une tentative visant à mobiliser le système de support du réseau social dans un effort de collaboration pour régler une crise émotionnelle. C'est une approche par objectifs et limitée dans le temps. Elle vise à mobiliser le réseau de façon à l'impliquer dans la création de nouvelles options et solutions pour régler une crise difficile." (Rueveni, 1979 : 17.)

Plus spécifiquement, une série d'études nous démontre le rôle important des réseaux sociaux dans l'atténuation ou la prévention des maladies physiques (Gore, 1978) et des problèmes d'adaptation psychologique (Nuckolls et al., 1972). Cela peut nous faire prendre d'autant plus conscience de la pertinence des écrits sur la situation spécifique du problème de l'intervention auprès des mourants. De plus, la méthodologie d'intervention réfère à un modèle existentiel (Daher, 1980).

Pour ne pas prolonger cette présentation, je joins l'Annexe II, qui décrit les différentes phases, tâches et buts de l'intervention de réseau familial. Cet outil de référence servira donc à l'élaboration du processus d'intervention des réseaux impliqués dans l'événement de la mort. 


\section{Conclusion}

L'intervention de réseau rend le milieu naturel et le milieu professionnel plus à l'écoute et plus actif aux besoins du mourant pour le sortir de l'isolement et le supporter dans les moments de crise. Ce modèle d'intervention vise à regrouper les énergies et à aider l'environnement à fournir un soutien de toutes sortes et vivre le processus du mourir comme une phase régulière de la vie d'une personne âgée.

Mais une aide est aussi apportée aux membres de la famille immédiate. Ceux-ci s'aident eux-mêmes lorsqu'ils sont mobilisés à la recherche de solutions. Pour des raisons multiples (expérience antérieure, formation professionnelle ou disponibilité), ils seront des atouts importants sur qui compter. D'autres se démobiliseront face à la maladie terminale par crainte et anxiété, ou par l'inexpérience de ces phénomènes. L'intervention portera donc tantôt sur des "exercices" pour crever l'anxiété et l'angoisse, pour faire surgir les énergies et pour les drainer et les coordonner. Car si chacun des membres de l'entourage immédiat est disponible à s'impliquer adéquatement dans la situation du mourir, il s'aidera à liquider la perte qu'il va subir et à cheminer lui-même dans ce processus de la mort d'un proche.

Je rappelle que les séquences d'activités proposées précédemment concernent le malade mais aussi les proches qui vivent avec lui. Peu d'insistance est mise sur cette dimension du phénomène de la mort dans le réseau (famille immédiate, parenté, amis, voisinage, etc.). Dans ces séquences d'activités, le réseau est d'abord aidant, bien sûr, et centré sur la personne aux prises avec la maladie terminale. Mais il sera d'autant plus efficace si le travailleur social veille à lui apporter de l'aide sur des points bien précis comme, entre autres:

- déculpabiliser les enfants éloignés en les faisant intervenir quand ils le pourront ;

- permettre à certains de décompresser par des activités de loisirs propres à leur goût ou intérêt et en maintenant une rotation des effectifs auprès du malade ;

- favoriser l'apprivoisement des membres de la famille immédiate à la réalité de la mort éventuelle par des lectures appropriées ou des entrevues individuelles avec eux. L'utilisation du groupe d'entraide pourrait être un moyen adéquat pour certaines personnes.

Finalement, ces étapes constituent une méthodologie d'intervention en situation de crise dans un modèle gérontologique. Un individu peut être mis dans une position vulnérable devant des événements subits et ceci le confronte à une situation de stress. Le 
manque de soutien situationnel pourra l'entraîner vers un état de déséquilibre et une crise possible. Il faut alors se retourner vers les personnes disponibles et significatives de l'entourage. Devant cette réalité de l'association de la vieillesse et de la mort, qui semble tant nous dérouter, il faut aller au-delà de ces apprivoisements individuels dans cette démarche professionnelle d'aide.

\section{Notes}

1 Les séquences d'activités du modèle d'intervention gérontologique ont été établies par $M$. Jacques Laforest, et utilisées par lui comme matériel du cours: "Intervention auprès des personnes âgées", École de service social, Université Laval.

${ }^{2}$ Ce réseau étant : la famille immédiate, la parenté, les amis et le voisinage du patient, ses connaissances.

\section{Références bibliographiques}

BURKE, R.J. et T. WEIR, "Giving and receiving help with work and non-work related problems", Journal of Business Administration, vol. 6, no 1, 1975 : 59-78.

CAREY-BÉlanger, Élaine et Christian Côté, "Les interventions brèves: huit ans après ", Service social, vol. 31, no 2-3, 1982: 240-289.

DAHER, Pierre, "Histoire d'une intervention de réseaux", Service social, vol. 29, no $3,1980: 284-303$.

GORE, S., "The effect of social support in moderating the health consequences of unemployment", Journal of Health and Social Behavior, vol. 19, no 2, 1978 : 157-165.

GuAY, J., L'intervenant professionnel face à l'aide naturelle, Chicoutimi, Gaëtan Morin, 1984.

LAFOREST, Jacques, notes du cours "Intervention auprès des personnes âgées", École de service social, Université Laval, 1983.

Nuckolls, K.B., J. CAssel et B.H. Kaplan, "Psychosocial assets, life crisis and the prognosis of pregnancy", American Journal of Epidemiology, vol. 95, 1972: 431-441.

PARAD, H.J. et G. CAPLAN, "A framework for studying families in crisis", dans: H.J. PARAD (éd.), Crisis Intervention: Selected Readings, New York, Family Service Association of America, 1965 : 53-74.

RUevenI, U., Networking Families in Crisis : Intervention Strategies With Families and Social Networks, New York, Human Sciences Press, 1979.

SaILlant, Francine, "Le cancer comme symbole de mort", Anthropologie et sociétés, vol. 6, no 3, 1982 : 91-104.

SPeCK, R.V. et C. Attneave, Family Networks, New York, Pantheon, 1973. 


\section{ANNEXE I}

Pour procéder à la lecture du tableau qui suit, il faut en considérer le contenu en faisant référence à une intervention en situation de crise, engendrée par un diagnostic de cancer. Nous en sommes alors à la phase curative de la maladie et non à sa phase terminale. Deux facteurs sont importants à retenir :

a) au début, il faut d'abord se demander s'il y a crise et, si c'est le cas, s'il s'agit d'une crise de développement (liée au passage d'une étape de vie à une autre) ou une crise accidentelle et subite (supposant une rupture importante dans la vie personnelle et sociale);

b) à la phase médiane, on devrait noter que l'intervention peut se situer à deux niveaux, à savoir favoriser les changements cognitifs et affectifs, et tendre vers l'apprentissage de nouveaux comportements. 
Intervention en situation de crise *

\begin{tabular}{|c|c|c|c|c|}
\hline Phase & Objectifs & Actions & Rôles & Techniques \\
\hline Début & $\begin{array}{l}\text { Rejoindre la personne dans } \\
\text { les plus brefs délais } \\
\text { Se centrer sur les facteurs } \\
\text { précipitants } \\
\text { Identifier le premier mode } \\
\text { de fonctionnement du } \\
\text { client } \\
\text { Engagement mutuel dans } \\
\text { l'exploration de la situation } \\
\text { Définir un guide de l'action }\end{array}$ & $\begin{array}{l}\text { Faire une prise de contact } \\
\text { rapide et adéquate } \\
\text { Établir la confiance et l'al- } \\
\text { liance avec le client } \\
\text { Établir un mini-contrat } \\
\text { d'entente mutuelle } \\
\text { Différencier comporte- } \\
\text { ment adéquat et inadéquat } \\
\text { Ouvrir sur le réseau et } \\
\text { les ressources }\end{array}$ & $\begin{array}{l}\text { Réduire la distance sociale } \\
\text { Reprendre le contrôle et } \\
\text { entrer en contact avec les } \\
\text { forces vitales du client } \\
\text { Être un allié dans la réso- } \\
\text { lution de problèmes } \\
\text { Rassurer le client en } \\
\text { l'encadrant } \\
\text { Décoder le non-verbal } \\
\text { Créer un climat de récipro- } \\
\text { cité } \\
\text { Développer l'espoir } \\
\text { Souligner les discordances }\end{array}$ & $\begin{array}{l}\text { Reformulation positive } \\
\text { Confrontation et intention } \\
\text { positive } \\
\text { Clarification, écoute } \\
\text { réflexive }\end{array}$ \\
\hline Milieu & $\begin{array}{l}\text { Maintenir l'attention sur la } \\
\text { situation à modifier et sur } \\
\text { l'objectif à atteindre } \\
\text { Centrer l'attention sur le } \\
\text { client, viser une conscience } \\
\text { cognitive de la situation }\end{array}$ & $\begin{array}{l}\text { Appliquer l'entente selon } \\
\text { l'ordre de priorité pré- } \\
\text { établi } \\
\text { Réaliser, par étapes, un } \\
\text { changement planifié } \\
\text { Consolider et synthétiser } \\
\text { les acquisitions, les appren- } \\
\text { tissages et les réussites du } \\
\text { client }\end{array}$ & $\begin{array}{l}\text { Assister, supporter et, si } \\
\text { nécessaire, stimuler les } \\
\text { énergies du système-client } \\
\text { Faire acquérir des compor- } \\
\text { tements nouveaux et } \\
\text { modifier des règles de vie } \\
\text { Encourager l'expression } \\
\text { des sentiments }\end{array}$ & $\begin{array}{l}\text { Utilisation consciente de soi } \\
\text { Partage de connaissances et } \\
\text { d'expériences de vie } \\
\text { Gestion du temps des } \\
\text { rencontres prévues }\end{array}$ \\
\hline
\end{tabular}




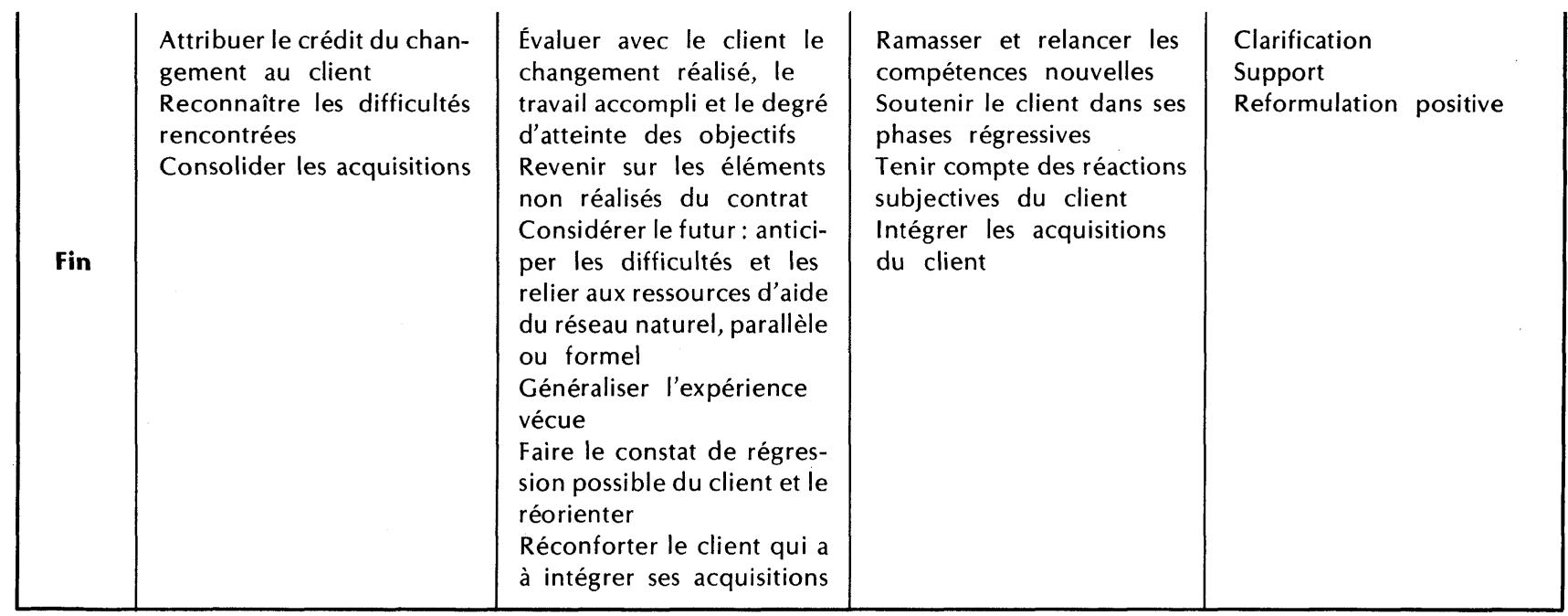

* Ce tableau a été réalisé à partir des écrits sur les phases d'intervention en situation de crise (Carey-Bélanger et Côté, 1982). 


\section{ANNEXE II}

\section{Intervention de réseau}

\begin{tabular}{|l|l|l|l|}
\hline \multicolumn{1}{|c|}{ Phase } & \multicolumn{1}{|c|}{ Tâches : famille/réseau } & Tâches : équipe d'intervention & \multicolumn{1}{c|}{ Buts } \\
\hline Pré-réseau & $\begin{array}{l}\text { Qui a le problème? } \\
\text {-penser en termes de réseau } \\
\text {-développer une confiance } \\
\text { dans les capacités du réseau }\end{array}$ & $\begin{array}{l}\text { Se dévoiler comme intervenant } \\
\text { Se présenter comme alternative } \\
\text { Faire le contrat (personnes à } \\
\text { impliquer, lieu à déterminer, } \\
\text { etc.) } \\
\text { Inviter ou solliciter des membres } \\
\text { du réseau }\end{array}$ & $\begin{array}{l}\text { Libérer et débloquer les énergies } \\
\text { Identifier les secrets et les coa- } \\
\text { litions }\end{array}$ \\
\hline $\begin{array}{l}\text { Intervention } \\
\text { 1. Retribalisation }\end{array}$ & $\begin{array}{l}\text { Prise de contact des membres du } \\
\text { réseau éparpillé les uns avec les } \\
\text { autres } \\
\text { Planification à partir des dispo- } \\
\text { nibilités de rencontres }\end{array}$ & $\begin{array}{l}\text { Faciliter les contacts entre les } \\
\text { membres du réseau } \\
\text { Réduire les tensions par des } \\
\text { simulations de rencontre }\end{array}$ & $\begin{array}{l}\text { Rendre le réseau visible } \\
\text { Renouer les liens entre les } \\
\text { membres }\end{array}$ \\
\hline 2. Polarisation & $\begin{array}{l}\text { Présentation du problème par la } \\
\text { famille: } \\
\text {-au niveau de la crise } \\
\text {-au niveau des attentes qu'ils } \\
\text { vivent }\end{array}$ & $\begin{array}{l}\text { Repérer des personnes actives } \\
\text { du réseau }\end{array}$ & $\begin{array}{l}\text { Aller chercher les émotions der- } \\
\text { rière les barrières structurelles }\end{array}$ \\
\hline
\end{tabular}




\begin{tabular}{|l|l|l|l|} 
3. Mobilisation & $\begin{array}{l}\text { Identification, par des membres } \\
\text { du réseau, des éléments de } \\
\text { solution }\end{array}$ & $\begin{array}{l}\text { Promouvoir les confrontations } \\
\text { directes (être un porte-parole) } \\
\text { Fournir du support individuel } \\
\text { Faciliter les communications }\end{array}$ & $\begin{array}{l}\text { Amener les énergies et res- } \\
\text { sources du réseau à trouver de } \\
\text { nouvelles solutions aux pro- } \\
\text { blèmes }\end{array}$ \\
\hline 4. Dépression & $\begin{array}{l}\text { Découragement collectif: le } \\
\text { problème est vu plus important } \\
\text { Prise de conscience de l'impor- } \\
\text { tance de la tâche }\end{array}$ & $\begin{array}{l}\text { Reconnaître les difficultés et } \\
\text { fournir des encouragements } \\
\text { au réseau }\end{array}$ & $\begin{array}{l}\text { Récupérer les sentiments positifs } \\
\text { de solidarité et supporter les } \\
\text { sentiments de découragement } \\
\text { Relancer l'espoir }\end{array}$ \\
\hline 5. Reprise & $\begin{array}{l}\text {-Les activités du réseau déter- } \\
\text { minent les solutions possibles et } \\
\text { réalisables }\end{array}$ & $\begin{array}{l}\text { Constituer des unités de travail } \\
\text { (interaction en petits groupes) }\end{array}$ & $\begin{array}{l}\text { Amener de nouvelles solutions } \\
\text { aux problèmes }\end{array}$ \\
\hline 6ibération \\
contentement & $\begin{array}{l}\text {-Développement de solutions } \\
\text { potentielles } \\
\text {-Planification de la poursuite de } \\
\text { l'implication des membres } \\
\text { du réseau }\end{array}$ & $\begin{array}{l}\text { Encourager le support aux } \\
\text { membres du groupe pour } \\
\text { l'établissement d'un plan } \\
\text { d'action }\end{array}$ & $\begin{array}{l}\text { Donner au réseau des senti- } \\
\text { ments de satisfaction et de } \\
\text { compétence } \\
\text { Renforcer la valeur de la tenue } \\
\text { des rencontres }\end{array}$ \\
\hline
\end{tabular}

* Cette synthèse est inspirée du matériel de cours du professeur Guy Bilodeau de l'École de service social de l'Université Laval. 